\title{
An attempt to study the Possible Inter-relationships amongst various Challenges Faced by Sports Industry in Indian Subcontinent
}

\author{
Lokik Aggarwal \\ Recventures Education services \\ Private Limited
}

\author{
Remica Aggarwal \\ MIT School of Education \& \\ Research \\ MIT Art, Design \& Technological \\ University Pune, India
}

\author{
Lakshay Aggarwal \\ Sociobuddy Technologies Private \\ Limited
}

\begin{abstract}
Present paper deals with recognizing various barriers or challenges for sports industry and under development of sports in India. The study is conducted in three stages. In the first stage, an exploratory study has been made and various barriers recognized through literature survey as well as internet / google search. In the second phase these recognized barriers were then scrutinized via a questionnaire survey where the respondents were the people from all age groups from Delhi -NCR region. Through the analysis the selected barriers have been further studied via the Interpretive Structural Modelling Methodology in the third stage .
\end{abstract}

Keywords

Sports Industry, ISM methodology

\section{INTRODUCTION}

Since 1970s , sports industry has assumed significant importance in a country's economy. With the globalization of public events, with sports participants, capital and labor, moving around the world, it has acquired a special place in capturing attention and giving recognition to a country. With global events such as FIFA, Olympic etc. capturing worldwide audiences, sports commodities have become a sought after things in today's economy. Since 1990s, there has been an significant increase in the number of players, moving to USA for training and participation in inter collegiate athletes in exchange of subsidized university education. Many countries already have or developing sport tourism and organizing events in recent years to drive and boost economy of the country [1]. The sport media tourism complex [1] driven sport is though multi-faceted but becoming more universal as cities such as Seoul, Beijing, Hongkong, New Delhi seeks to host major global events[2] .

During 1990s and early 200s, media corporation spended at unprecedented level at sporting coverage an team league ownership particularly as television companies became global entities. It is evident that sports have emerged out of twentieth century with significant structural changes . direct media ownership of clubs and franchises, leagues and events and tours become common by 2000 as leading media companies have attempted to corner global communication markets $[3,4,5]$. However in the process of global sport, there are also a number of countries which lag behind due to inappropriate infrastructure or paucity of support from its government [ $3,6]$.

Present research work focuses on exploring such challenges and barriers which hamper the growth of a country's sports industry. The challenges have been studies with a view for developing country such as India. The paper is organized as follows. Section 2 deals with literature review. Section 3 deals with ISM methodology. Section 4 deals with application of methodology to case example. Section 5 concludes the paper.

\section{LITERATURE REVIEW}

Sports have been popular for many, many years. Yet with the rise in popularity also comes the rise of challenges and adversity [3]. Various challenges have been explored via google scholar internet explorer, Research gate search engine etc. A questionnaire was constructed and the responses received and as per the responses the major challenges faced by Indian sports industry ${ }^{1}$ has been as follows :

1. Problem in attracting the millennial generation (PAMG): With the technological advances, people are more used to watch the sports from the comforts of their home . Toppings on the cake has been that the professional sporting groups such as the NBA and NFL have also made it easier through mobile applications and their own TV stations (NFL Network, for example).

2. Problem in technological integration with social media (PTISM) : Many fans connect with their favorite teams through social media, which puts pressure on teams to constantly keep updating their Facebook timelines and Twitter feeds. Thus, if teams are not proactive with social media and do not have the capability of enticing fans with modern technology, fans will simply not follow them nor go to games in person.

3. Meeting the challenge of game day experience (MCGDE): This includes pregame shows, halftime entertainment, and postgame celebrations. Not to mention, coming up with in-game experiences are just as important, whether it be the t-shirt gun, kiss cam, chants, or anything else they can come up with. This is becoming quite important these days to attract and retain viewers.

4. Coping up with the challenge of tackling negative stories about sportsmen (CNSS): One challenge the sports industry will always face is the hyper focus on negative stories surrounding athletes. There is always a section of society / viewers who thirst for such scandals and negative headlines . 
5. Coping the pressure of predicting future trends (PPFT) : While the future is nearly impossible to predict, it might be worthwhile for the sports industry to study trends and attempt to predict what will be popular in the future.

6. Infrastructure challenges / poor sports ecosystem (IC): Poor infrastructure often imposes a major challenge before the sports industry. Poor performance in major international events such as Olympics against countries such as Cuba, Croatia etc. shows the lack of world-class infrastructure and the inadequate support of the government . Reasons could be insufficient private sector funding; restrictive guidelines for availing government grants for sports infrastructure; poor asset utilization and inefficient monetization planning. An initiative has been taken by Union cabinet by revamping the Khelo India programme at a cost of Rs.1,756 crore for the period 2017-18 to 2019-20 ${ }^{1}$ which would impact the entire sports ecosystem, including infrastructure, community sports, talent identification, coaching for excellence, competition structure and sports economy.

7. Poor/ weak support to sportswomen / negative image of sports and sportswomen (WSW): It has been observed by many researchers [ 7] the women's attitude towards sports and the myths that exist in the society regarding sports participation hinders women to join sports. People believes that participation in sports and physical activities may create hurdle in marriage as well as child bearing of the women sportsperson. Government is launching new schemes to motivate women to join sports and providing financial assistance to the recognized National Sports Federations (NSFs) for supporting girls/women's exposure, training and participation at national/ international level.

8. Inadequate trainers (IT) : Indian sports industry do face a crunch of skilled trainers . a nationwide shortage of professionally trained and qualified coaches limits the quality of training for aspiring athletes . A lack of executive-level sports management training opportunities inhibits the development of trained professionals to manage leagues and major sporting events. In order to overcome this, for example for their athletes for the upcoming 2020 Olympics, the government approved the appointment of foreign coaches and supporting staff.

9. Corruption \& Mismanagement of sports authorities (CSA) : Corruption has become synonymous with sports administration in India. Whether it is the most popular cricket or hockey or weightlifting, most of the sports authorities in India have come under attack due to corruption charges. Controversies surrounding 2010 Commonwealth Games has also dented the image of sports administrators in India.

10. Social and economic inequalities (SEI) : Social and economic inequalities have a negative impact on the Indian sport. Denial of access to sports infrastructure due to poverty, concentration of stadiums and other sports avenues only in cities, lack of encouragement to girls to participate in sports, etc, have impaired the development of a positive sports culture in the country.

11. Lack of effective policies (LEP) : For the development of any sector, formulation and execution of an effective policy is a must. This is true for sports also. Till date, the sports policy planning and implementation is centralized in the country due to the paucity of resources and the expertise by the State and local governments. Moreover, the absence of a separate ministry of sports at the union level reflects the apathy towards sports.

12. Budgetary constraints / Meagre allocation of resources/ weak incentives (BC) : Compared to other developed and developing countries, allocation of financial resources is meagre in India. In the Union Budget 2017-18, Rs 1943 crore allocated for sports. While it is Rs 450 crore higher than the previous year, it is much below than the around Rs 9000 crore spent annually by the UK for the sports sector. scholarships and endowments are fraught with bureaucratic red tape or lack of process transparency. post-career support for athletes or coaches is lacking and further limits professional participation. Further there is little or no postretirement support in the sector such as pension schemes or support for launching private ventures.

13. The policy and regulatory environment (RE) : As with other sectors, public sector capacity to manage sport is limited at both the central and state government levels. Funding is slow to mobilize. Challenges in navigating and engaging with multiple government bodies can delay effective collaboration.

14. Skills and education gaps (SEG) : These gaps exists at all levels of professionalism . At the grassroots, low integration of sports into school curriculums and a lack of proper physical education and sports training are major factors limiting school sports participation and physical literacy among children. At the elite level, limited higher education and research in sports sciences (including nutrition, psychology, medicine and sports education) hinders efforts to maximize player performance.

15. Cultural / Language barriers ${ }^{2}$ (C/LB) amongst sports persons : There are cultural barriers to grassroots participation in sport in India. There is a commonly held view that sport is an unnecessary diversion from children's studies rather than an integral part of education and development and a potential career pathway. Further, language is a common barrier. Not every Indian sportsperson is well versed with English or Hindi in communications. Appointing foreign coaches will always makes our athletes smarter in their preparations and skills provided they understand the language and able to communicate with each other confidently

16. Weak government supporting partnership programs (WGSPP): India is a price-sensitive market and revenue models need to be adjusted compared to developed western markets. Many of our competitor countries have recently replicated 
and surpassed Australia's innovation and investment in the 1980s and 1990s. Prioritizing India on the Australian Government sporting agenda and expanding micro programs to support development through sport could alleviate the matter.

\section{INTERPRETIVE STRUCTURAL MODELLING METHODOLOGY}

Suggested by [43], the technique has been used widely to develop a map of the relationships between the many elements in the form of a hierarchy graph. Group's judgement decides whether and how the items are related. Steps in applying an ISM methodology involves identification of relevant elements and then establishing contextual relationship amongst them taking elements pairwise. Further to this a SSIM i.e. the structural self- interaction matrix has been developed followed by initial and then final reachability matrix [Warfield (1994)] . A dependence and driving element diagram and a diagraph can be obtained afterwards to see the hierarchical relationship amongst the relevant elements .

\section{DEVELOPMENT OF ISM MODEL}

In this section, ISM model is developed for studying the interrelationships amongst various challenges for Indian sports industry .

Some 16 challenges have been identified viz. Media / Audience related challenges which includes Problem in attracting the millennial generation (PAMG) to watch the show; Problem in technological integration with social media (PTISM) ; Meeting the challenge of game day experience (MCGDE) ; Challenge of tackling with negative stories about sportsmen (CNSS) and Pressure of predicting future trends (PPFT) . Then there are infrastructure related challenges which includes Infrastructure challenges / poor sports ecosystem (IC) ; Poor/ weak support to sportswomen / negative image of sports and sportswomen (WSW) ; Inadequate trainers (IT) ; Skills and education gaps (SEG) ; Cultural / Language barriers ${ }^{2}$ (C/LB) amongst sports persons. Then there are Socio - economic challenges which include Corruption \& Mismanagement of sports authorities (CSA); Social and economic inequalities (SEI) ; Lack of effective policies (LEP) ; Budgetary constraints / Meagre allocation of resources / weak incentives (BC) ; The policy and regulatory environment (RE) ; Weak government supporting partnership programs (WGSPP).
Explanation : If you are facing challenge in attracting negative stories about sportsmen then there could be a problem in attracting millennials as one section of millennials is very much interested in hearing about scandals . Corruption and mismanagement could be a aftermath of weak infrastructure . Negative image of sports women could be a result of poor social media awareness . Poor social media could also lead to cultural or language barriers and weak government supporting partnership programs or vice versa Legal and Regulatory environment could lead to weak government supporting programs, poor support to women , corruption and mismanagement. Budgetary constraints could be the prime reason for almost all the challenges be it skills and education gaps, be it language barriers, be it hiring of qualified trainer etc. SEI could lead to PPFT . Policy or regulatory environment may lead to lack of effective policies, corruption or mismanagement of sports authority etc. Social and economic inequalities could create corruption and mismanagement of authorities and vice versa. PAMG affects PTISM and vice versa and that could affect meeting the challenge of game day experience. Infrastructure changes as well as negative image and stories about sportsmen could create problem in meeting game day experience. Weak government supported partnership programs could lead to barrier in gathering masses as these collaboration programs have a high command in international market . Cultural and education barriers could be one of the reason for getting inadequate trainers. Cultural and language barriers could be the reason for not receiving appropriate education, weak skills . Budgetary constraints could aggravate cultural and language barriers as people are unable to upgrade themselves .

\subsection{Construction of Structural Self - Interaction Matrix (SSIM)}

This matrix gives the pair-wise relationship between two variables i.e. $i$ and $j$ based on VAXO. SSIM has been presented below in Fig 1.

\subsection{Construction of Initial Reachability Matrix and final reachability matrix}

The SSIM has been converted in to a binary matrix called the initial reachability matrix shown in fig. 2 by substituting V, A, $\mathrm{X}, \mathrm{O}$ by 1 or 0 as per the case. After incorporating the transitivity, the final reachability matrix is shown below in the Fig 3.

Fig 1: SSIM matrix for pair wise relationship amongst barriers

\begin{tabular}{|c|c|c|c|c|c|c|c|c|c|c|c|c|c|c|c|c|c|}
\hline S.no. & $\begin{array}{l}\text { Success } \\
\text { factors }\end{array}$ & $\mathbf{1}$ & $\mathbf{2}$ & $\mathbf{3}$ & $\mathbf{4}$ & $\mathbf{5}$ & $\mathbf{6}$ & $\mathbf{7}$ & $\mathbf{8}$ & $\mathbf{9}$ & $\mathbf{1 0}$ & $\mathbf{1 1}$ & $\mathbf{1 2}$ & $\mathbf{1 3}$ & $\mathbf{1 4}$ & $\mathbf{1 5}$ & $\mathbf{1 6}$ \\
\hline & & PAMG & PTISM & MCGDE & CNSS & PPFT & IC & WSW & IT & CSA & SEI & LEP & BC & RE & SEG & C/LB & WGSPP \\
\hline 1 & PAMG & & X & A & A & V & A & A & O & A & O & A & A & A & O & A & A \\
\hline 2 & PTISM & & & V & V & V & A & V & O & A & O & A & A & O & O & V & X \\
\hline 3 & MCGDE & & & & X & V & A & A & O & A & O & A & A & A & O & O & O \\
\hline 4 & CNSS & & & & & V & A & A & O & A & O & A & A & A & O & A & O \\
\hline 5 & PPFT & & & & & & A & A & O & A & A & A & A & A & O & A & O \\
\hline 6 & IC & & & & & & & V & V & A & A & X & A & A & V & V & A \\
\hline 7 & WSW & & & & & & & A & A & O & A & A & A & A & A & O \\
\hline 8 & IT & & & & & & & & & A & O & A & A & A & V & A & O \\
\hline 9 & CSA & & & & & & & & & A & X & V & X & X & V & O & V \\
\hline
\end{tabular}




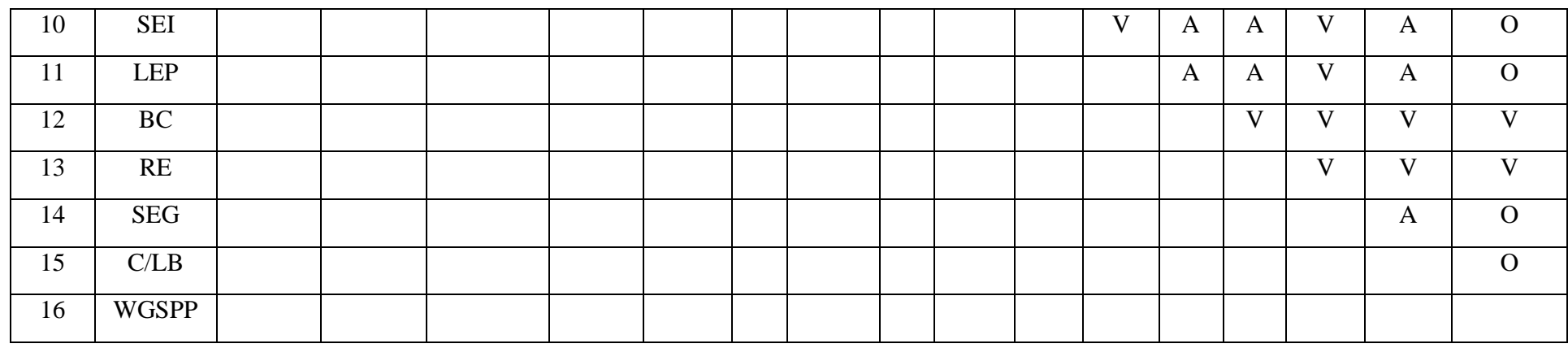

Fig 2: Initial reachability matrix

\begin{tabular}{|c|c|c|c|c|c|c|c|c|c|c|c|c|c|c|c|c|c|}
\hline S.no. & $\begin{array}{l}\text { Success } \\
\text { factors }\end{array}$ & 1 & 2 & 3 & 4 & 5 & 6 & 7 & 8 & 9 & 10 & 11 & 12 & 13 & 14 & 15 & 16 \\
\hline & & PAMG & PTISM & MCGDE & CNSS & PPFT & IC & WSW & IT & $\mathrm{CSA}$ & SEI & LEP & $\mathrm{BC}$ & $\mathrm{RE}$ & SEG & C/LB & WGSPP \\
\hline 2 & PTISM & 1 & 1 & 1 & 1 & 1 & 0 & 1 & 0 & 0 & 0 & 0 & 0 & 0 & 0 & 1 & 1 \\
\hline 3 & MCGDE & 1 & 0 & 1 & 1 & 1 & 0 & 0 & 0 & 0 & 0 & 0 & 0 & 0 & 0 & 0 & 0 \\
\hline 5 & PPFT & 0 & 0 & 0 & 0 & 1 & 0 & 0 & 0 & 0 & 0 & 0 & 0 & 0 & 0 & 0 & 0 \\
\hline 6 & IC & 1 & 1 & 1 & 1 & 1 & 1 & 1 & 1 & 0 & 0 & 1 & 0 & 0 & 1 & 1 & 0 \\
\hline 7 & WSW & 1 & 0 & 1 & 1 & 1 & 0 & 1 & 0 & 0 & 0 & 0 & 0 & 0 & 0 & 0 & 0 \\
\hline 8 & IT & 0 & 0 & 0 & 0 & 0 & 0 & 1 & 1 & 0 & 0 & 0 & 0 & 0 & 1 & 0 & 0 \\
\hline 12 & $\mathrm{BC}$ & 1 & 1 & 1 & 1 & 1 & 1 & 1 & 1 & 1 & 1 & 1 & 1 & 1 & 1 & 1 & 1 \\
\hline 13 & $\mathrm{RE}$ & 1 & 1 & 1 & 1 & 1 & 1 & 1 & 1 & 1 & 1 & 1 & 0 & 1 & 1 & 1 & 1 \\
\hline 14 & SEG & 0 & 1 & 0 & 0 & 0 & 0 & 1 & 0 & 0 & 0 & 0 & 0 & 0 & 1 & 0 & 0 \\
\hline 15 & $\mathrm{C} / \mathrm{LB}$ & 1 & 1 & 0 & 1 & 1 & 0 & 1 & 1 & 0 & 1 & 1 & 0 & 0 & 1 & 1 & 0 \\
\hline 16 & WGSPP & 1 & 1 & 0 & 0 & 0 & 1 & 0 & 0 & 0 & 0 & 0 & 0 & 0 & 0 & 0 & 1 \\
\hline
\end{tabular}

Fig 3 : Final reachability matrix

\begin{tabular}{|c|c|c|c|c|c|c|c|c|c|c|c|c|c|c|c|c|c|c|}
\hline S.no. & $\begin{array}{l}\text { Success } \\
\text { factors }\end{array}$ & $\mathbf{1}$ & $\mathbf{2}$ & $\mathbf{3}$ & $\mathbf{4}$ & $\mathbf{5}$ & $\mathbf{6}$ & $\mathbf{7}$ & $\mathbf{8}$ & $\mathbf{9}$ & $\mathbf{1 0}$ & $\mathbf{1 1}$ & $\mathbf{1 2}$ & $\mathbf{1 3}$ & $\mathbf{1 4}$ & $\mathbf{1 5}$ & $\mathbf{1 6}$ & $\mathbf{D . P}$ \\
\hline & & PAMG & PTISM & MCGDE & CNSS & PPFT & IC & WSW & IT & CSA & SEI & LEP & BC & RE & SEG & C/LB & WGSPP & D.P \\
\hline 1 & PAMG & 1 & 1 & 1 & 1 & 1 & 0 & 1 & 0 & 0 & 0 & 0 & 0 & 0 & 0 & 1 & 1 & 8 \\
\hline 2 & PTISM & 1 & 1 & 1 & 1 & 1 & 1 & 1 & 1 & 0 & 1 & 0 & 0 & 0 & 1 & 1 & 1 & 12 \\
\hline 3 & MCGDE & 1 & 1 & 1 & 1 & 1 & 0 & 0 & 0 & 0 & 0 & 0 & 0 & 0 & 0 & 0 & 0 & 5 \\
\hline 4 & CNSS & 1 & 1 & 1 & 1 & 1 & 0 & 0 & 0 & 0 & 0 & 0 & 0 & 0 & 0 & 0 & 0 & 5 \\
\hline 5 & PPFT & 0 & 0 & 0 & 0 & 1 & 0 & 0 & 0 & 0 & 0 & 0 & 0 & 0 & 0 & 0 & 0 & 1 \\
\hline 6 & IC & 1 & 1 & 1 & 1 & 1 & 1 & 1 & 1 & 0 & 1 & 1 & 0 & 0 & 1 & 1 & 1 & 13 \\
\hline 7 & WSW & 1 & 1 & 1 & 1 & 1 & 0 & 1 & 0 & 0 & 0 & 0 & 0 & 0 & 0 & 0 & 0 & 6 \\
\hline 8 & IT & 1 & 1 & 1 & 1 & 1 & 0 & 1 & 1 & 0 & 0 & 0 & 0 & 0 & 1 & 0 & 0 & 8 \\
\hline 9 & CSA & 1 & 1 & 1 & 1 & 1 & 1 & 1 & 1 & 1 & 1 & 1 & 1 & 1 & 1 & 1 & 1 & 0 \\
\hline
\end{tabular}




\begin{tabular}{|c|c|c|c|c|c|c|c|c|c|c|c|c|c|c|c|c|c|c|}
\hline 10 & SEI & 1 & 1 & 1 & 1 & 1 & 1 & 1 & 1 & 1 & 1 & 1 & 0 & 0 & 1 & 1 & 0 & 13 \\
\hline 11 & LEP & 1 & 1 & 1 & 1 & 1 & 1 & 1 & 1 & 0 & 0 & 1 & 0 & 0 & 1 & 1 & 1 & 12 \\
\hline 12 & BC & 1 & 1 & 1 & 1 & 1 & 1 & 1 & 1 & 1 & 1 & 1 & 1 & 1 & 1 & 1 & 1 & 0 \\
\hline 13 & RE & 1 & 1 & 1 & 1 & 1 & 1 & 1 & 1 & 1 & 1 & 1 & 1 & 1 & 1 & 1 & 1 & 0 \\
\hline 14 & SEG & 1 & 1 & 1 & 1 & 1 & 0 & 1 & 0 & 0 & 0 & 0 & 0 & 0 & 1 & 0 & 0 & 7 \\
\hline 15 & C/LB & 1 & 1 & 1 & 1 & 1 & 1 & 1 & 1 & 1 & 1 & 1 & 0 & 0 & 1 & 1 & 0 & 13 \\
\hline 16 & WGSPP & 1 & 1 & 1 & 1 & 1 & 1 & 1 & 1 & 0 & 1 & 1 & 0 & 0 & 1 & 1 & 1 & 13 \\
\hline & De.P & 15 & 15 & 15 & 15 & 16 & 9 & 13 & 10 & 5 & 8 & 8 & 3 & 3 & 11 & 10 & 8 & \\
\hline
\end{tabular}

D.P : Driving power ; De.P : dependence power

\subsection{Level Partition}

Table 2 : Iteration I

\begin{tabular}{|c|c|c|c|c|}
\hline $\begin{array}{c}\text { S.No } \\
\text {. }\end{array}$ & $\begin{array}{c}\text { Reachability } \\
\text { set }\end{array}$ & $\begin{array}{c}\text { Antecedent } \\
\text { set }\end{array}$ & $\begin{array}{l}\text { Intersecti } \\
\text { on set }\end{array}$ & $\begin{array}{c}\text { Iteratio } \\
\mathbf{n} /\end{array}$ \\
\hline 1. & 5 & $\begin{array}{c}1,2,3,4,5,6,7,8,91 \\
0,11,12 \\
13,14,15,16\end{array}$ & 5 & \multirow{12}{*}{$\mathbf{I}$} \\
\hline 2. & $1,2,3,4,5$ & $\begin{array}{c}1,2,3,4,6,7,8,9 \\
10,11,12,13 \\
14,15,16\end{array}$ & $1,2,3,4$ & \\
\hline 3. & $1,2,3,4,5,7$ & $\begin{array}{c}1,2,6,7,8,9,10 \\
11,12,13,14 \\
15,16\end{array}$ & $1,2,7$ & \\
\hline 4. & $\begin{array}{c}1,2,3,4,5,7 \\
14\end{array}$ & $\begin{array}{c}2,6,8,9,10,11,12 \\
13,14,15,16\end{array}$ & $2,7,14$ & \\
\hline 5. & $\begin{array}{c}1,2,3,4,5,7,8 \\
14\end{array}$ & $\begin{array}{c}2,6,8,9,10,11,12 \\
13,15,16\end{array}$ & 2,8 & \\
\hline 6. & $\begin{array}{c}1,2,3,4,5,7 \\
14,15\end{array}$ & $\begin{array}{c}2,6,9,10,11,12,13 \\
, 15,16\end{array}$ & 2,15 & \\
\hline 7. & $\begin{array}{c}1,2,3,4,5,6,7, \\
8,14,15\end{array}$ & $\begin{array}{c}2,6,9,10,11,12,13 \\
, 14,15,16\end{array}$ & $2,6,14,15$ & \\
\hline 8. & $\begin{array}{c}1,2,3,4,5,6,7 \\
8,14,15,16\end{array}$ & $2,6,9,11,12,13,16$ & $2,6,16$ & \\
\hline 9. & $\begin{array}{c}1,2,3,4,5,6,7, \\
8,11,14,15\end{array}$ & $\begin{array}{c}6,9,10, \\
11,12,13,16\end{array}$ & $6,11,16$ & \\
\hline 10. & $\begin{array}{c}1,2,3,4,5,6,7 \\
8,10,11,14\end{array}$ & $\begin{array}{c}2,6,9,10,12,13 \\
16\end{array}$ & $2,6,9,10$ & \\
\hline 11. & $\begin{array}{c}1,2,3,4,5,6,7 \\
8,9 \\
11,14,15,16\end{array}$ & $9,12,13$ & 9 & \\
\hline 12. & $\begin{array}{c}1,2,3,4,5,6,7, \\
8,9,10,11, \\
12,13,14,15, \\
16\end{array}$ & $9,12,13$ & $9,12,13$ & \\
\hline
\end{tabular}

From the final reachability matrix, reachability and final antecedent set for each factor are found. The element for which the reachability and intersection sets are same are the top-level element in the ISM hierarchy. After the identification of top level element, it is separated out from the other elements and the process continues for next level of elements. Reachability set, antecedent set, intersection set along with different level for elements have been shown below in table 2 to table 11 .

Table 3 : Iteration II

\begin{tabular}{|c|c|c|c|c|}
\hline $\begin{array}{c}\text { S.No } \\
\text {. }\end{array}$ & $\begin{array}{c}\text { Reachability } \\
\text { set }\end{array}$ & Antecedent set & $\begin{array}{c}\text { Intersection } \\
\text { set }\end{array}$ & $\begin{array}{l}\text { Itera } \\
\text { tion }\end{array}$ \\
\hline 2. & $1,2,3,4$ & $\begin{array}{c}1,2,3,4,6,7,8,9 \\
10,11,12,13 \\
14,15,16\end{array}$ & $1,2,3,4$ & \multirow{11}{*}{ II } \\
\hline 3. & $1,2,3,4,7$ & $\begin{array}{c}1,2,6,7,8,9,10 \\
11,12,13,14,15,1 \\
6\end{array}$ & $1,2,7$ & \\
\hline 4. & $1,2,3,4,7,14$ & $\begin{array}{c}2,6,8,9,10,11,12 \\
13,14,15,16\end{array}$ & $2,7,14$ & \\
\hline 5. & $\begin{array}{c}1,2,3,4,7,8,1 \\
4\end{array}$ & $\begin{array}{c}2,6,8,9,10,11,12 \\
13,15,16\end{array}$ & 2,8 & \\
\hline 6. & $\begin{array}{c}1,2,3,4,7 \\
14,15\end{array}$ & $\begin{array}{c}2,6,9,10,11,12 \\
13,15,16\end{array}$ & 2,15 & \\
\hline 7. & $\begin{array}{c}1,2,3,4,6,7,8 \\
14,15\end{array}$ & $\begin{array}{c}2,6,9,10,11,12 \\
13,14,15,16\end{array}$ & $2,6,14,15$ & \\
\hline 8. & $\begin{array}{c}1,2,3,4,6,7,8 \\
14,15,16\end{array}$ & $\begin{array}{c}2,6,9,11,12,13 \\
16\end{array}$ & $2,6,16$ & \\
\hline 9. & $\begin{array}{c}1,2,3,4,6,7,8 \\
11,14,15\end{array}$ & $\begin{array}{c}\text { 6,9,10, } \\
11,12,13,16\end{array}$ & $6,11,16$ & \\
\hline 10. & $\begin{array}{c}1,2,3,4,6,7,8 \\
10,11,14\end{array}$ & $\begin{array}{c}2,6,9,10,12,13 \\
16\end{array}$ & $2,6,9,10$ & \\
\hline 11. & $\begin{array}{c}1,2,3,4,6,7,8 \\
9,11,14 \\
15,16\end{array}$ & $9,12,13$ & 9 & \\
\hline 12. & $\begin{array}{c}1,2,3,4,6,7,8 \\
9,10,11,12,1 \\
3,14,15,16\end{array}$ & $9,12,13$ & $9,12,13$ & \\
\hline
\end{tabular}


Table 4 : Iteration III

\begin{tabular}{|c|c|c|c|c|}
\hline $\begin{array}{l}\text { Sr. } \\
\text { No. }\end{array}$ & $\begin{array}{c}\text { Reachability } \\
\text { set }\end{array}$ & $\begin{array}{c}\text { Antecedent } \\
\text { set }\end{array}$ & $\begin{array}{c}\text { Intersection } \\
\text { set }\end{array}$ & $\begin{array}{l}\text { Itera } \\
\text { tion }\end{array}$ \\
\hline 3. & 7 & $\begin{array}{c}6,7,8,9,10,11 \\
12,13,14,15 \\
16\end{array}$ & 7 & \multirow{10}{*}{ III } \\
\hline 4. & 7,14 & $\begin{array}{c}6,8,9,10,11,1 \\
2,13,14,15,1 \\
6\end{array}$ & 7,14 & \\
\hline 5. & $7,8,14$ & $\begin{array}{c}6,8,9,10,11,1 \\
2,13,15,16\end{array}$ & 8 & \\
\hline 6. & $7,14,15$ & $\begin{array}{c}2,6,9,10,11,1 \\
2,13,15,16\end{array}$ & 15 & \\
\hline 7. & $6,7,8,14,15$ & $\begin{array}{c}6,9,10,11,12 \\
13,14,15,16\end{array}$ & $6,14,15$ & \\
\hline 8. & $6,7,8,14,15,16$ & $\begin{array}{c}6,9,11,12,13 \\
16\end{array}$ & 6,16 & \\
\hline 9. & $6,7,8,11,14,15$ & $\begin{array}{c}6,9,10 \\
11,12,13,16\end{array}$ & $6,11,16$ & \\
\hline 10. & $6,7,8,10,11,14$ & $\begin{array}{c}6,9,10,12,13 \\
16\end{array}$ & $6,9,10$ & \\
\hline 11. & $\begin{array}{c}6,7,8,9,11,14,1 \\
5,16\end{array}$ & $9,12,13$ & 9 & \\
\hline 12. & $\begin{array}{c}6,7,8,9,10,11,1 \\
2,13,14,15 \\
16\end{array}$ & $9,12,13$ & $9,12,13$ & \\
\hline
\end{tabular}

Table 6 : Iteration IV

\begin{tabular}{|c|c|c|c|c|}
\hline $\begin{array}{c}\text { S.No } \\
.\end{array}$ & $\begin{array}{c}\text { Reachability } \\
\text { set }\end{array}$ & Antecedent set & $\begin{array}{l}\text { Intersecti } \\
\text { on set }\end{array}$ & $\begin{array}{c}\text { Iterat } \\
\text { ion/ } \\
\text { Level } \\
\mathbf{s}\end{array}$ \\
\hline 4. & 14 & $\begin{array}{c}6,8,9,10,11,12 \\
13,14,15,16\end{array}$ & 14 & \multirow{9}{*}{ IV } \\
\hline 5. & 8,14 & $\begin{array}{c}6,8,9,10,11,12 \\
13,15,16\end{array}$ & 8 & \\
\hline 6. & 14,15 & $\begin{array}{c}2,6,9,10,11,12 \\
13,15,16\end{array}$ & 15 & \\
\hline 7. & $6,8,14,15$ & $\begin{array}{c}6,9,10,11,12 \\
13,14,15,16\end{array}$ & $6,14,15$ & \\
\hline 8. & $6,8,14,15,16$ & $6,9,11,12,13,16$ & 6,16 & \\
\hline 9. & $6,8,11,14,15$ & $\begin{array}{c}6,9,10, \\
11,12,13,16\end{array}$ & $6,11,16$ & \\
\hline 10 . & $6,8,10,11,14$ & $\begin{array}{c}6,9,10,12,13 \\
16\end{array}$ & $6,9,10$ & \\
\hline 11. & $\begin{array}{c}6,8,9,11,14,1 \\
5,16\end{array}$ & $9,12,13$ & 9 & \\
\hline 12. & $\begin{array}{c}6,8,9,10,11,1 \\
2,13, \\
14,15,16\end{array}$ & $9,12,13$ & $9,12,13$ & \\
\hline
\end{tabular}

Table 7 : Iteration V

\begin{tabular}{|c|c|c|c|c|}
\hline $\begin{array}{l}\text { Sr. } \\
\text { No. }\end{array}$ & $\begin{array}{c}\text { Reachability } \\
\text { set }\end{array}$ & $\begin{array}{c}\text { Antecedent } \\
\text { set }\end{array}$ & $\begin{array}{c}\text { Intersection } \\
\text { set }\end{array}$ & $\begin{array}{l}\text { Itera } \\
\text { tion }\end{array}$ \\
\hline 5. & 8 & $\begin{array}{c}6,8,9,10,11,1 \\
2,13,15,16\end{array}$ & 8 & \multirow{8}{*}{ V } \\
\hline 6. & 15 & $\begin{array}{c}2,6,9,10,11,1 \\
2,13,15,16\end{array}$ & 15 & \\
\hline 7. & $6,8,15$ & $\begin{array}{c}6,9,10,11,12 \\
13,15,16\end{array}$ & 6,15 & \\
\hline 8. & $6,8,15,16$ & $\begin{array}{c}6,9,11,12,13 \\
16\end{array}$ & 6,16 & \\
\hline 9. & $6,8,11,15$ & $\begin{array}{c}6,9,10 \\
11,12,13,16\end{array}$ & $6,11,16$ & \\
\hline 10. & $6,8,10,11$ & $\begin{array}{c}6,9,10,12,13 \\
16\end{array}$ & $6,9,10$ & \\
\hline 11. & $6,8,9,11,15,16$ & $9,12,13$ & 9 & \\
\hline 12. & $\begin{array}{c}6,8,9,10,11,12 \\
13,15,16\end{array}$ & $9,12,13$ & $9,12,13$ & \\
\hline
\end{tabular}

Table 8 : Iteration VI

\begin{tabular}{|c|c|c|c|c|}
\hline $\begin{array}{l}\text { Sr. } \\
\text { No. }\end{array}$ & $\begin{array}{c}\text { Reachability } \\
\text { set }\end{array}$ & Antecedent set & $\begin{array}{c}\text { Intersection } \\
\text { set }\end{array}$ & $\begin{array}{l}\text { Itera } \\
\text { tion }\end{array}$ \\
\hline 5. & 8 & $\begin{array}{c}6,8,9,10,11,12 \\
13,16\end{array}$ & 8 & \multirow{7}{*}{ VI } \\
\hline 7. & 6,8 & $\begin{array}{c}6,9,10,11,12 \\
13,16\end{array}$ & 6 & \\
\hline 8. & $6,8,16$ & $\begin{array}{c}6,9,11,12,13 \\
16\end{array}$ & 6,16 & \\
\hline 9. & $6,8,11$ & $\begin{array}{c}6,9,10, \\
11,12,13,16\end{array}$ & $6,11,16$ & \\
\hline 10. & $6,8,10,11$ & $\begin{array}{c}6,9,10,12,13 \\
16\end{array}$ & $6,9,10$ & \\
\hline 11. & $6,8,9,11,16$ & $9,12,13$ & 9 & \\
\hline 12. & $\begin{array}{c}6,8,9,10,11,12 \\
13,16\end{array}$ & $9,12,13$ & $9,12,13$ & \\
\hline
\end{tabular}

Table 9 : Iteration VII

\begin{tabular}{|c|c|c|c|c|}
\hline $\begin{array}{l}\text { Sr. } \\
\text { No. }\end{array}$ & $\begin{array}{c}\text { Reachability } \\
\text { set }\end{array}$ & $\begin{array}{c}\text { Antecedent } \\
\text { set }\end{array}$ & $\begin{array}{c}\text { Intersection } \\
\text { set }\end{array}$ & $\begin{array}{l}\text { Itera } \\
\text { tion }\end{array}$ \\
\hline 7. & 6 & $\begin{array}{c}6,9,10,11,12 \\
13,16\end{array}$ & 6 & \multirow{6}{*}{ VII } \\
\hline 8. & 6,16 & $\begin{array}{c}6,9,11,12,13, \\
16\end{array}$ & 6,16 & \\
\hline 9. & 6,11 & $\begin{array}{c}6,9,10, \\
11,12,13,16\end{array}$ & 6,11 & \\
\hline 10. & $6,10,11$ & $\begin{array}{c}6,9,10,12,13 \\
16\end{array}$ & $6,9,10$ & \\
\hline 11. & $6,9,11,16$ & $9,12,13$ & 9 & \\
\hline 12. & $\begin{array}{c}6,9,10,11,12,1 \\
3,16\end{array}$ & $9,12,13$ & $9,12,13$ & \\
\hline
\end{tabular}


Table 10 : Iteration VIII

\begin{tabular}{|c|c|c|c|c|}
\hline $\begin{array}{l}\text { Sr. } \\
\text { No. }\end{array}$ & $\begin{array}{c}\text { Reachability } \\
\text { set }\end{array}$ & $\begin{array}{c}\text { Antecedent } \\
\text { set }\end{array}$ & $\begin{array}{c}\text { Intersection } \\
\text { set }\end{array}$ & $\begin{array}{l}\text { Itera } \\
\text { tion }\end{array}$ \\
\hline 8. & 16 & $\begin{array}{c}9,11,12,13 \\
16\end{array}$ & 16 & \multirow{5}{*}{ VIII } \\
\hline 9. & 11 & $\begin{array}{c}9,10, \\
11,12,13,16\end{array}$ & 11 & \\
\hline 10. & 10,11 & $\begin{array}{c}9,10,12,13 \\
16\end{array}$ & 9,10 & \\
\hline 11. & $9,11,16$ & $9,12,13$ & 9 & \\
\hline 12. & $\begin{array}{c}9,10,11,12,13 \\
16\end{array}$ & $9,12,13$ & $9,12,13$ & \\
\hline
\end{tabular}

Table 11 : Iteration IX

\begin{tabular}{|c|c|c|c|c|}
\hline $\begin{array}{l}\text { Sr. } \\
\text { No. }\end{array}$ & $\begin{array}{c}\text { Reachability } \\
\text { set }\end{array}$ & $\begin{array}{c}\text { Antecedent } \\
\text { set }\end{array}$ & $\begin{array}{c}\text { Intersection } \\
\text { set }\end{array}$ & $\begin{array}{l}\text { Itera } \\
\text { tion }\end{array}$ \\
\hline 8. & 16 & $9,12,13,16$ & 16 & \\
\hline 10. & 10 & $\begin{array}{c}9,10,12,13 \\
16\end{array}$ & 9,10 & IX \\
\hline 11. & 9,16 & $9,12,13$ & 9 & \\
\hline 12. & $9,10,12,13,16$ & $9,12,13$ & $9,12,13$ & \\
\hline
\end{tabular}

Table 12 : Iteration $\mathrm{X}$

\begin{tabular}{|c|c|c|c|c|}
\hline $\begin{array}{c}\text { Sr. } \\
\text { No. }\end{array}$ & $\begin{array}{c}\text { Reachability } \\
\text { set }\end{array}$ & $\begin{array}{c}\text { Antecedent } \\
\text { set }\end{array}$ & $\begin{array}{c}\text { Intersection } \\
\text { set }\end{array}$ & $\begin{array}{c}\text { Itera } \\
\text { tion }\end{array}$ \\
\hline 8. & $\mathbf{1 6}$ & $9,12,13,16$ & 16 & \\
\cline { 1 - 4 } 11. & 9,16 & $9,12,13$ & 9 & \multirow{2}{*}{$\mathbf{X}$} \\
\hline 12. & $9,12,13,16$ & $9,12,13$ & $9,12,13$ & \\
\hline
\end{tabular}

Table 12 : Iteration XI

\begin{tabular}{|c|c|c|c|c|}
\hline $\begin{array}{c}\text { Sr. } \\
\text { No. }\end{array}$ & $\begin{array}{c}\text { Reachability } \\
\text { set }\end{array}$ & $\begin{array}{c}\text { Antecedent } \\
\text { set }\end{array}$ & $\begin{array}{c}\text { Intersection } \\
\text { set }\end{array}$ & $\begin{array}{c}\text { Itera } \\
\text { tion }\end{array}$ \\
\hline 11. & 9 & $9,12,13$ & 9 & XI \\
\hline 12. & $\mathbf{9 , 1 2 , 1 3}$ & $9,12,13$ & $9,12,13$ & \\
\hline
\end{tabular}

\subsection{Classification of factors}

The critical success factors described earlier are classified in to four clusters viz. autonomous factor, dependent factors, linkage factors and independent factors (mentioned in Table XIII below). As it can be seen that CLC is an autonomous criteria. Criteria DTC, DOC, LTR, EC, MO , MS are drivers . Criteria such as REL, LTQP , IOIVE, LALC, ABB , LTP are dependent criteria. Criteria TE and BRS are linkage criteria.

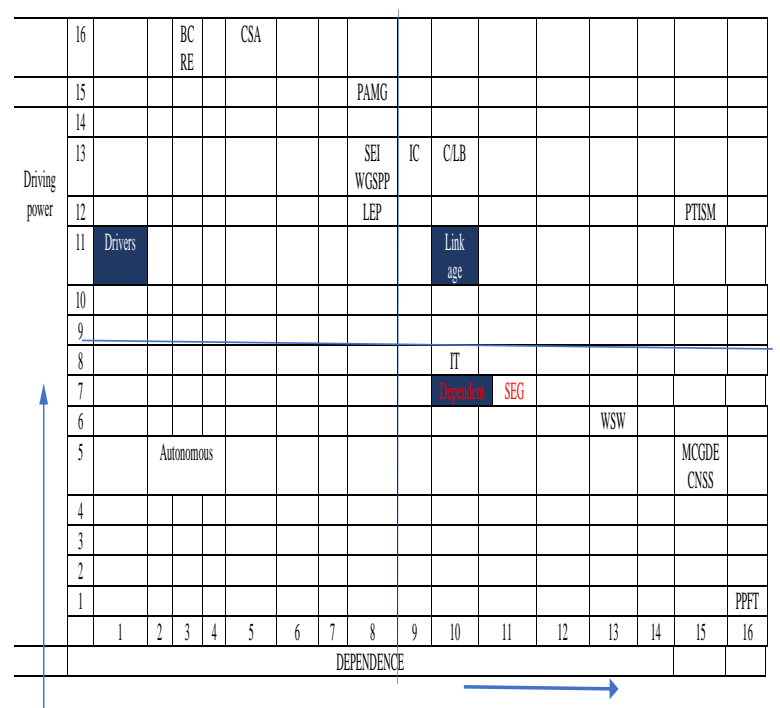

Fig . 4: Driving power and dependence diagram

\subsection{ISM model}

An ISM model is developed ( as shown in fig. 5 below ) after arranging the elements as per their interaction or dependence relationships.

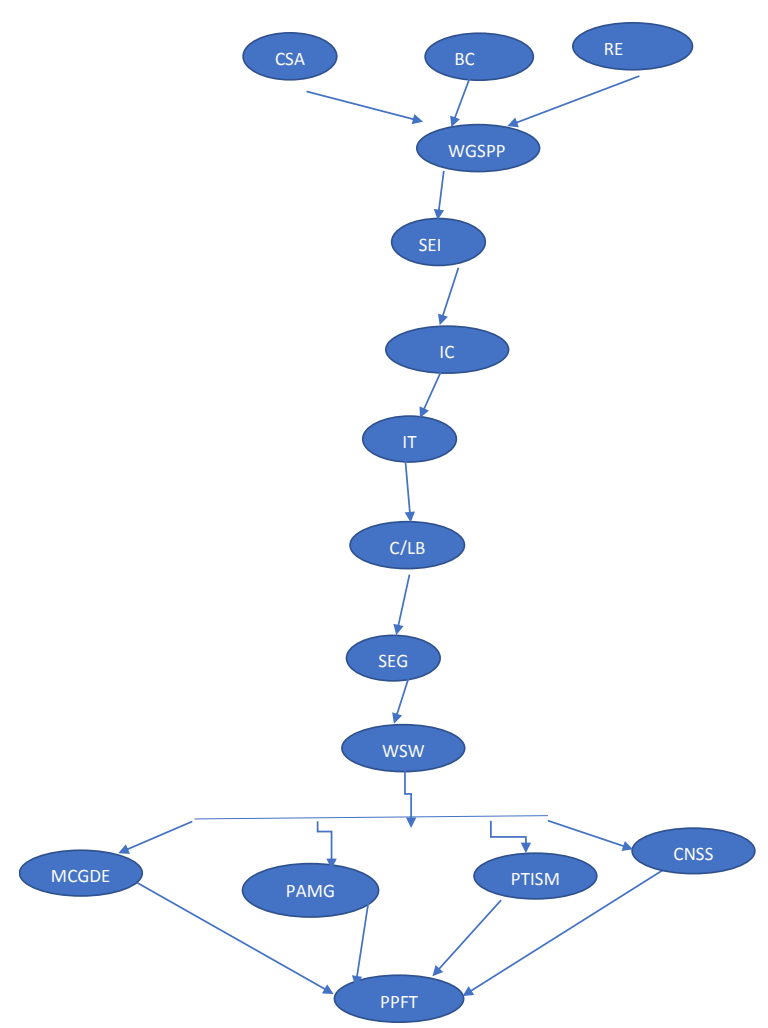

Fig 5: ISM diagraph

\section{CONCLUSIONS}

Through the analysis the selected barriers have been further studied via the Interpretive Structural Modelling Methodology. It has been found that BC, RE, CSA, SEI , WGSPP etc are the drivers whereas IC , C/LB , PTISM are the linkage criteria. SEG, IT, WSW , MCGDE, CNSS , PPFT etc are dependent criteria . 


\section{REFERENCES}

[1] Nauright , J. 2015. Beyond the sports media tourism complex : an agenda for transforming sports “, Journal of international council of sports science and physical education , 68(5), 13-19.

[2] Nadvi , K. Lunds, Thomsen , P. , Xue , H. and Khara , N. 2011. Playing against China : global value chains and labor standards in the international sports goods industry . Global networks , 11(3), 334-354.

[3] Zhang, J.J. , Kim , E. , Marstromartino , B. , Qian , T.Y., Nauright , J. 2018. The sports industry in growing economy : critical issues and challenges, International Journal of sports marketing and sponsorship, 19(2), 110126.

[4] Pitts , B.G. and Zhang, J.J. 2016 . Introduction : the WASM foundation stone. Global sport management : contemporary issues and inquiries. Rouledge London , 13-17.

[5] Sage, G. 2011. Globalizing sports : How organisations , corporations, media and politics are changing sports, Rouledge, london.

[6] Zhang, J.J., Pitts , B.G., Kim , E. 2017. Introduction : sport marketing in a globalized market place. Contemporary sport marketing : global perspectives , Rouledge London , 3-20.

[7] Lata , K. , Lata, S., Kumar, M. 2015. Access to basic sports facilities in India. A survey of 12 universities in Haryana, Munich, GRIN Verlag, https://www.grin.com/document/306329.

[8] Warfield, J.N. 1974. Developing interconnection matrices in structural modeling. IEEE Transactions on Systems, Man, and Cybernetics, (1), 81-87. 